\title{
Mesons in NA61/SHINE
}

\author{
Antoni Marcinek ${ }^{1, *}$ for the NA61/SHINE Collaboration \\ ${ }^{1}$ Institute of Nuclear Physics, Polish Academy of Sciences, PL-31342 Kraków, Poland
}

\begin{abstract}
This contribution presents a selection of recent results on meson production from the NA61/SHINE experiment. The results include spectra of $\phi$ mesons in $p+p$ collisions at 40,80 and $158 \mathrm{GeV} / c$ beam momenta, as well as $\pi^{ \pm}, K^{ \pm}, \rho^{0}, \omega$ and $K^{* 0}$ mesons in $\pi^{-}+\mathrm{C}$ collisions at $158 \mathrm{GeV} / c$ and $350 \mathrm{GeV} / c$. They are compared with predictions of several models. A spectacular failure of considered models is observed for $\phi, \rho^{0}$ and $K^{* 0}$ production. Also a very peculiar system size dependence of the longitudinal evolution of $\phi$ production, contrasting with all other measured hadrons, is shown. Finally, preliminary results on spectator-induced electromagnetic effects in $\pi^{ \pm}$production in $\mathrm{Ar}+\mathrm{Sc}$ at $150 \mathrm{~A} \mathrm{GeV} / \mathrm{c}$ are presented, bringing information on space-time evolution of the hot and dense matter created in the collision.
\end{abstract}

\section{Introduction}

The NA61/SHINE experiment at the CERN SPS [1] pursues a two-fold physics programme: (1) measurements of hadron spectra in hadron-nucleus collisions for neutrino and cosmic rays experiments; and (2) study of strongly interacting matter via a two-dimensional beam momentum and system size scan, including correlations, fluctuations and hadron spectra. Reactions measured within both parts of the programme are sketched in Fig. 1. This article discusses only a selection of recent results from this rich collection. The important results on pion and kaon production obtained from the $2 \mathrm{D}$ scan as well as future measurements of open charm mesons are tackled in separate contributions [2, 3].

\section{$2 \phi(1020)$ meson in $p+p$ collisions}

Motivation for the measurement of $\phi(1020)$ meson production in $p+p$ collisions is twofold. On one hand, it may serve to constrain hadron production models. On the other hand, it may be used as reference for $\mathrm{Pb}+\mathrm{Pb}$ measurements at the same energies to conclude about strangeness-related phenomena in heavy ion collisions.

In Fig. 2 transverse momentum spectra of $\phi$ mesons in $p+p$ collisions at $80 \mathrm{GeV} / c$ and $40 \mathrm{GeV} / c$ beam momenta, in rapidity bins, are compared to three models: Epos 1.99 [4, 5], PyтнIa 6.4.28 [6] and URQMD 3.4 [7, 8]. These models are normalized to the integral of the data in each rapidity bin, so that the shape of the spectrum can be compared. It is visible that Pyтнia describes well the $p_{T}$ spectrum shape, while the spectra from URQMD are too hard and those from Epos too soft. These are the first ever differential measurements of $\phi$ production at these energies.

\footnotetext{
*e-mail: antoni.marcinek@gmail.com
} 


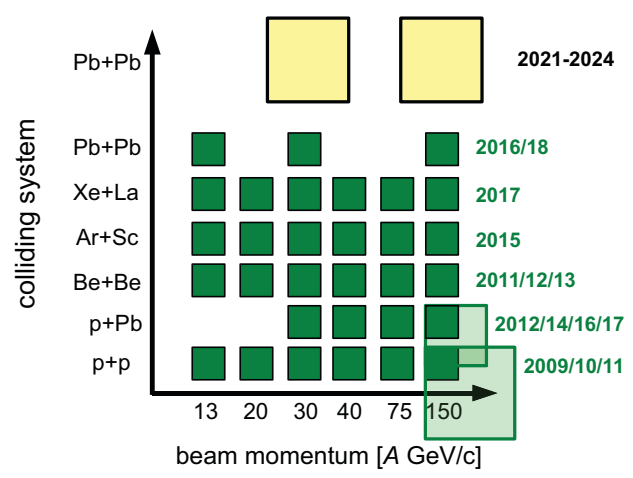

$\square \square$ recorded

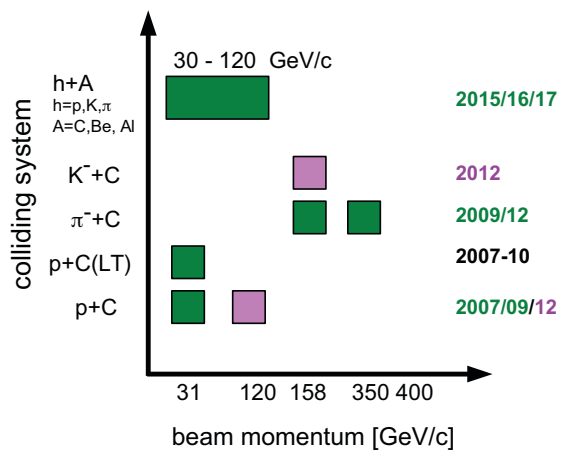

proposed

Figure 1: Reactions collected by NA61/SHINE and proposed after CERN Long Shutdown 2 within both parts of the physics programme. Size of squares visualizes amount of data with typical sample size of few million events. Worth noting are measurements with meson beams.

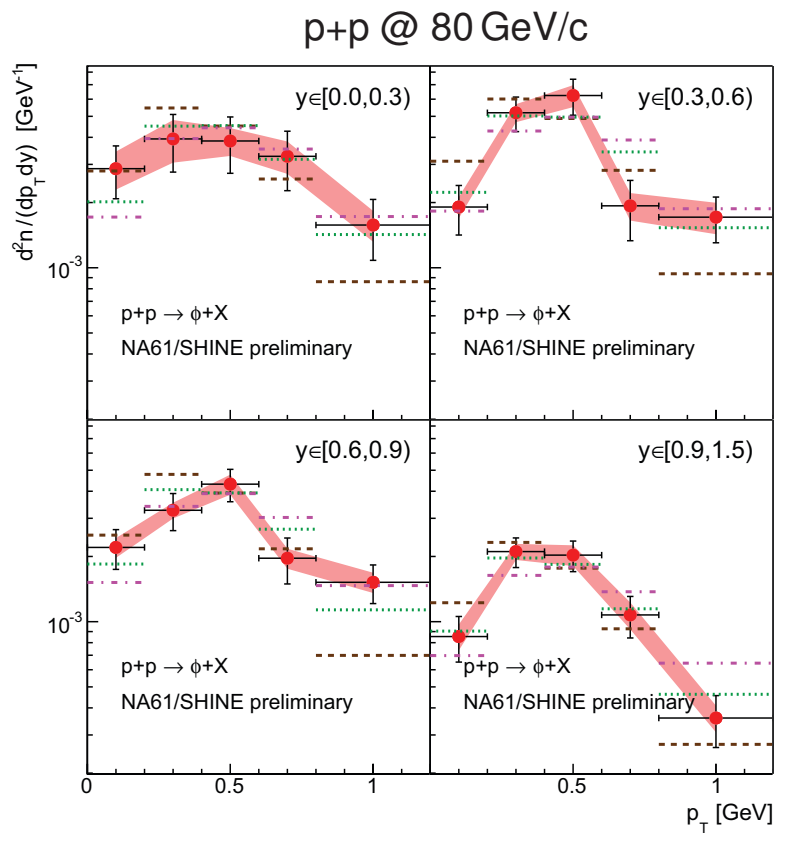

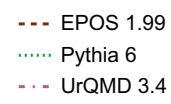

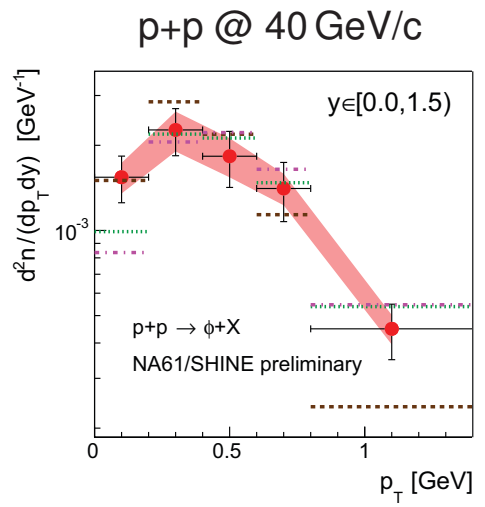

Figure 2: Transverse momentum spectra of $\phi$ mesons in $p+p$ collisions at $80 \mathrm{GeV} / c$ and $40 \mathrm{GeV} / c$ beam momenta in rapidity bins with statistical (vertical lines) and systematic (bands) uncertainties. Horizontal lines give $p_{T}$ bin sizes. Regarding models see text. 

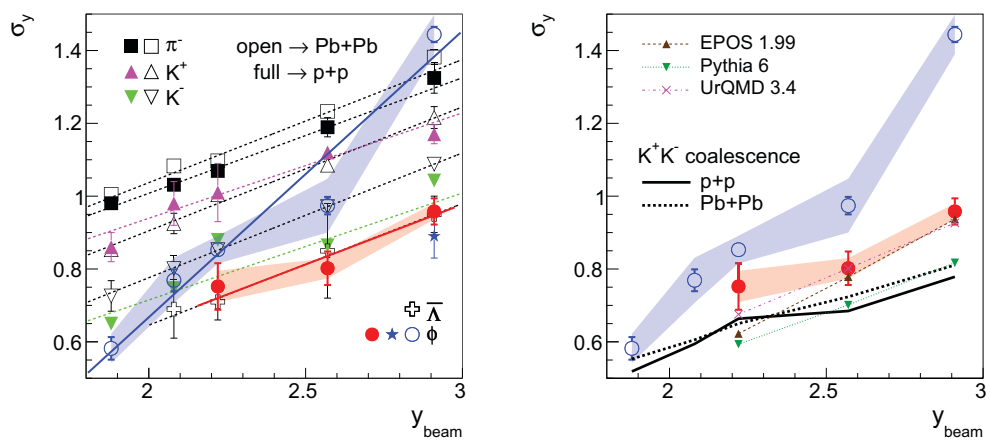

Figure 3: Left: Widths of rapidity distributions of various particles in $p+p$ (full symbols) and central $\mathrm{Pb}+\mathrm{Pb}$ (open symbols) collisions as a function of beam rapidity, with statistical (vertical lines) and systematic (bands) uncertainties. Full red circles are results of this analysis, the blue star is the NA49 $p+p$ measurement [10], other $p+p$ points come from NA61/ SHINE [11, 12]. $\mathrm{Pb}+\mathrm{Pb}$ data are from NA49 [13-16]. Lines are fitted to points to guide the eye. Right: Comparison of widths of rapidity distributions of $\phi$ mesons with expectations from kaon coalescence and models (see text).

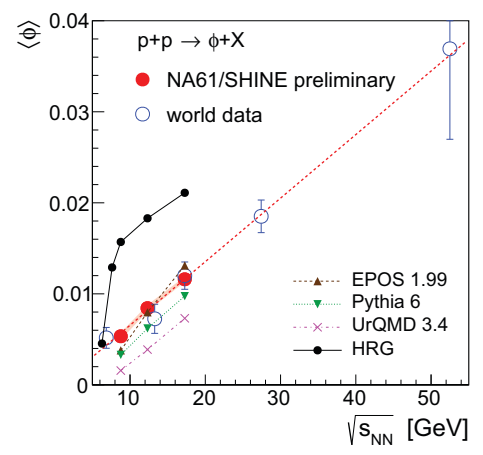

Figure 4. Energy dependence of total yields of $\phi$ mesons in $p+p$ collisions. World data come from Refs. [10, 17-20]. Total uncertainties are shown; for preliminary NA61/SHINE results total uncertainties are smaller than markers. Red dashed line is fitted to guide the eye. Regarding models see text.

Fig. 3 presents widths $\sigma_{\mathrm{y}}$ of rapidity distributions of $\phi$ mesons [9] and various other particles in $p+p$ and central $\mathrm{Pb}+\mathrm{Pb}$ collisions [10-16] as a function of beam rapidity $\mathrm{y}_{\text {beam }}$ in the centre-of-mass frame. It is apparent that all particles in both colliding systems, except $\phi$ in $\mathrm{Pb}+\mathrm{Pb}$ follow the same trend. This non-trivial system size dependence for $\phi$ mesons, contrasting with other hadrons, calls for investigation in other systems measured within the 2D scan of NA61/SHINE (Fig. 1, left).

The widths are also compared to predictions of models, showing that PyTHIA produces too narrow $\phi$ rapidity spectra, while both Epos and URQMD provide widths comparable to experimental data. Moreover, Fig. 3 demonstrates that the experimental results are not compatible with the simple picture of $\phi$ production through $K^{+} K^{-}$coalescence [13].

The energy dependence of total $\phi$ yields in $p+p$ interactions is displayed in Fig. 4. It is evident that NA61/SHINE results are consistent with the world data [10, 17-20], but provide superior accuracy. Comparing to models, it is visible that only Epos describes the data reasonably well, while others fail with URQMD underestimating and HRG [21] overestimating the yield by about a factor of 2 . 

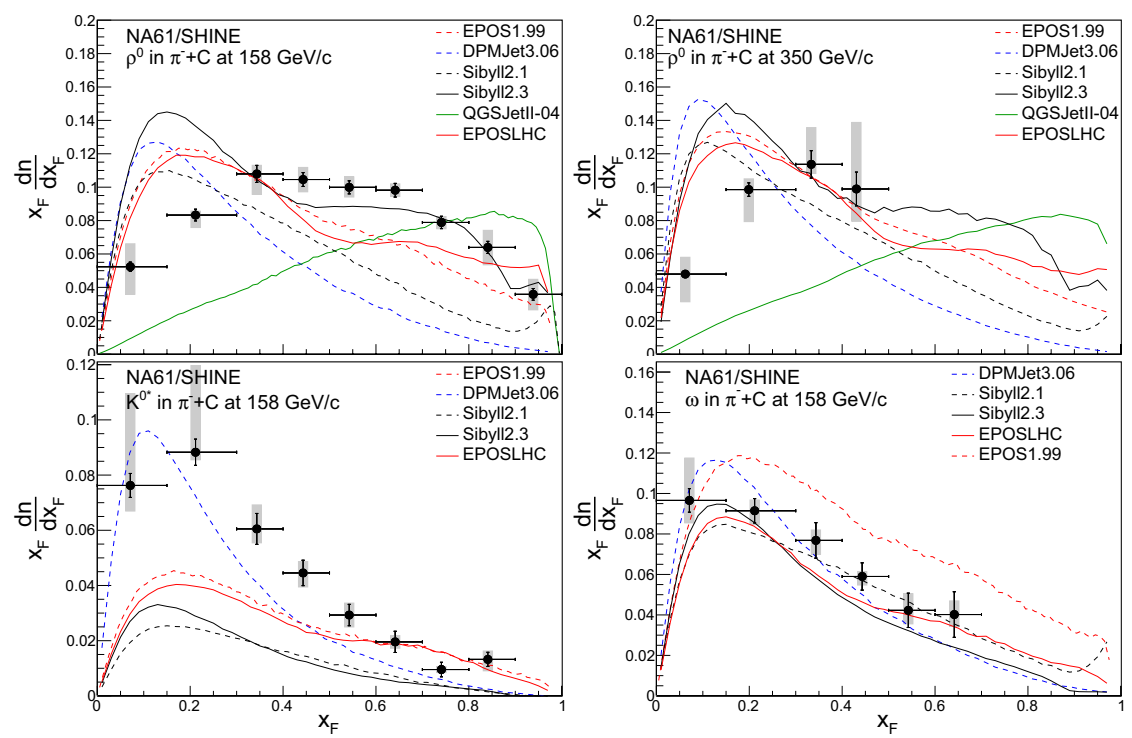

Figure 5: Spectra of $\rho^{0}, \omega$ and $K^{* 0}$ mesons in $\pi^{-}+\mathrm{C}$ collisions at beam momenta of $158 \mathrm{GeV} / c$ and $350 \mathrm{GeV} / c$ as a function of $x_{\mathrm{F}}$. The statistical uncertainties are represented by bars and the systematic ones by grey bands [22]. Regarding models see text.

\section{Production of mesons in $\pi^{-}+\mathbf{C}$ collisions}

Indirect measurements of high energy cosmic rays through Extensive Air Showers (EAS) require air shower simulations to be interpreted. These simulations rely to a large extent on a correct modelling of hadron-air interactions that occur during the shower development [23]. Especially the pion-nucleus collisions data are important for models tuning, as these are the most abundant hadronic interactions occurring in EAS.

Spectra of $\rho^{0}, \omega$ and $K^{* 0}$ [22], as well as $\pi^{ \pm}$and $K^{ \pm}$mesons in $\pi^{-}+\mathrm{C}$ collisions at beam momenta of $158 \mathrm{GeV} / c$ and $350 \mathrm{GeV} / c$ are presented in Fig. 5 and Fig. 6. These results are compared to predictions of several models used in EAS simulations: QGSJET II-04 [24], Epos 1.99 [25], DPMJet 3.06 [26], Sibyll 2.1 [27], Sibyll 2.3 [28] and Epos LHC [29]. It is apparent that while at least some of these models are able to describe $\omega$ and $\pi^{ \pm}$production, all fail for $\rho^{0}$ and kaons. This shows that the new data constitute an important input to tune hadron production models necessary to understand cosmic-ray air showers.

\section{Spectator-induced EM effects}

The spectator-induced electromagnetic distortion of charged pion ratio was first observed in peripheral $\mathrm{Pb}+\mathrm{Pb}$ collisions at $158 \mathrm{~A} \mathrm{GeV} / c$ beam momentum [30] (Fig. 7, left). This charge asymmetry is caused by the final state electromagnetic repulsion (attraction) of positive (negative) pions by the spectator system. It is the largest for pions moving at spectator rapidity.

Now the effect is measured also in $\mathrm{Ar}+\mathrm{Sc}$ collisions at $150 \mathrm{~A} \mathrm{GeV} / c$ beam momentum. This is the first observation of this distortion in small systems at the SPS. While for peripheral $\mathrm{Pb}+\mathrm{Pb}$ (spectator charge about 70 e.u.) the effect was large, in intermediate centrality $\mathrm{Ar}+\mathrm{Sc}$ (Fig. 7, middle; spectator charge about 8 e.u.) it is still visible and large enough to break isospin symmetry. Interestingly, for the case of central $\mathrm{Ar}+\mathrm{Sc}$ (Fig. 7, right) some 

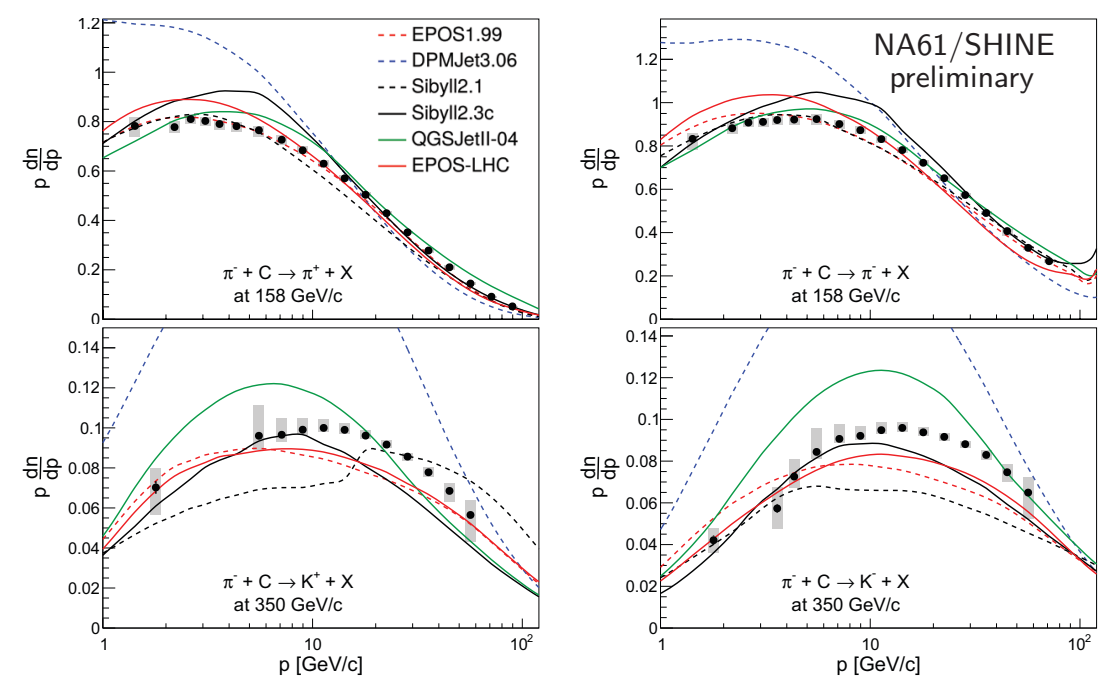

Figure 6: Spectra of $\pi^{ \pm}$and $K^{ \pm}$mesons in $\pi^{-}+\mathrm{C}$ collisions at beam momenta of $158 \mathrm{GeV} / c$ and $350 \mathrm{GeV} / c$ as a function of momentum $p$. The statistical uncertainties are represented by bars and the systematic ones by grey bands. Regarding models see text.
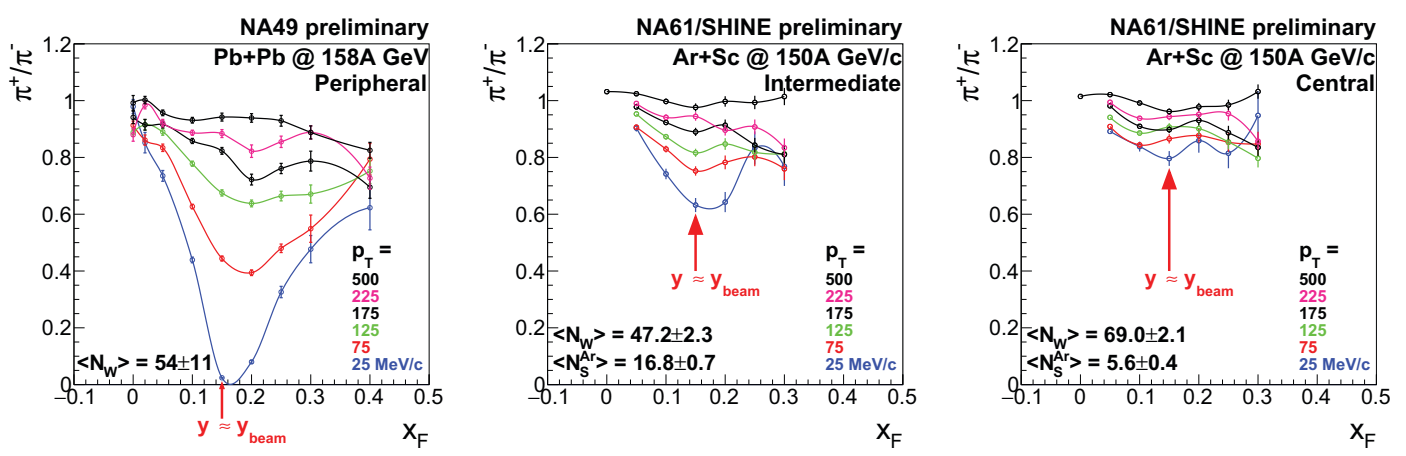

Figure 7: The $\pi^{+} / \pi^{-}$ratio as a function of $x_{\mathrm{F}}$ for several values of transverse momentum in peripheral $\mathrm{Pb}+\mathrm{Pb}$ [30], intermediate centrality $\mathrm{Ar}+\mathrm{Sc}$ and central $\mathrm{Ar}+\mathrm{Sc}$ collisions at the top CERN SPS beam momentum. Only statistical uncertainties are shown.

lowering of the ratio at beam rapidity can still be observed, suggesting a shadow of the EM distortion even for such a low spectator charge (about 3 e.u.).

It is known that the spectator-induced EM effect brings information on the space-time evolution of the reaction [31]. The new results allow to obtain such information for $\mathrm{Ar}+\mathrm{Sc}$ collisions along with an insight into energy transfers in the spectator system [32].

\section{Summary}

A selection of recent results on meson production measured by the NA61/SHINE experiment has been presented. The first results on differential multiplicities of $\phi$ mesons in $p+p$ collisions at CERN SPS energies show superior accuracy compared to other measurements of $\phi$ 
production in $p+p$ collisions and emphasize the peculiarity of the system size dependence of the width of rapidity distributions of $\phi$ mesons. The spectra of $\pi^{ \pm}, K^{ \pm}, \rho^{0}, \omega$ and $K^{* 0}$ mesons in $\pi^{-}+\mathrm{C}$ provide important input to tune hadron production models necessary to understand cosmic-ray air showers. The spectator-induced EM effects in charged pion production give insight to space-time properties of the system created in heavy ion collisions.

This work was supported by the National Science Centre, Poland (grant numbers: 2014/14/E/ST2/00018, 2015/18/M/ST2/00125) and the Foundation for Polish Science - MPD program, co-financed by the European Union within the European Regional Development Fund.

\section{Bibliography}

[1] N. Abgrall et al. (NA61/SHINE), JINST 9, P06005 (2014)

[2] D. Larsen, The onsets of deconfinement and fireball of NA61/SHINE, this proceedings

[3] A. Merzlaya, Open charm measurements at CERN SPS energies with the new Vertex Detector of the NA61/SHINE experiment, this proceedings

[4] K. Werner, F. Liu, T. Pierog, Phys. Rev. C 74, 044902 (2006)

[5] T. Pierog, K. Werner, Nucl. Phys. B (Proc. Suppl.) 196, 102 (2009)

[6] T. Sjöstrand, S. Mrenna, P. Skands, J. High Energy Phys. 05, 026 (2006)

[7] S. Bass et al., Prog. Part. Nucl. Phys. 41, 255 (1998)

[8] M. Bleicher et al., J. Phys. G 25, 1859 (1999)

[9] A. Marcinek, EPJ Web Conf. 182, 02082 (2018)

[10] S. Afanasiev et al. (NA49), Phys. Lett. B 491, 59 (2000)

[11] N. Abgrall et al. (NA61/SHINE), Eur. Phys. J. C 74, 2794 (2014)

[12] A. Aduszkiewicz et al. (NA61/SHINE), Eur. Phys. J. C 77, 671 (2017)

[13] C. Alt et al. (NA49), Phys. Rev. C 78, 044907 (2008)

[14] S.V. Afanasiev et al. (NA49), Phys. Rev. C 66, 054902 (2002)

[15] C. Alt et al. (NA49), Phys. Rev. C 77, 024903 (2008)

[16] T. Anticic et al. (NA49), Phys. Rev. Lett. 93, 022302 (2004)

[17] V. Blobel et al., Phys. Lett. B 59, 88 (1975)

[18] C. Daum et al. (ACCMOR), Nucl. Phys. B 186, 205 (1981)

[19] D. Drijard et al., Z. Phys. C 9, 293 (1981)

[20] M. Aguilar-Benitez et al. (LEBC-EHS), Z. Phys. C 50, 405 (1991)

[21] V. Vovchenko, V.V. Begun, M.I. Gorenstein, Phys. Rev. C 93, 064906 (2016)

[22] A. Aduszkiewicz et al. (NA61/SHINE), Eur. Phys. J. C77, 626 (2017)

[23] R. Engel, D. Heck, T. Pierog, Ann. Rev. Nucl. Part. Sci. 61, 467 (2011)

[24] S. Ostapchenko, Phys.Rev. D83, 014018 (2011)

[25] T. Pierog, K. Werner, Phys. Rev. Lett. 101, 171101 (2008)

[26] S. Roesler, R. Engel, J. Ranft, in Proc. MC2000 (2000), pp. 1033-1038

[27] E.J. Ahn et al., Phys.Rev. D80, 094003 (2009)

[28] F. Riehn et al., PoS (ICRC2015), 558 (2015)

[29] T. Pierog et al., Phys. Rev. C92, 034906 (2015)

[30] A. Rybicki, Acta Phys. Polon. B42, 867 (2011)

[31] A. Rybicki, A. Szczurek, Phys. Rev. C75, 054903 (2007)

[32] A. Rybicki, this proceedings 